\title{
ELABORACIÓN DE NANOPARTÍCULAS DE ORO EN HIDROGELES TERMOSENSITIVOS
}

\author{
Jesús Tito Gutarra ${ }^{\mathrm{a}}$, Juan Carlos Rueda ${ }^{\mathrm{a}}$, Alberto Corzo ${ }^{\mathrm{b}}$
}

\begin{abstract}
RESUMEN
En esta investigación se estudió la factibilidad de elaborar nanopartículas de oro (NP-Au) dentro de hidrogeles termosensitivos hechos a partir de N-isopropilacrilamida (NiPAAm) y macromonómeros (MMH) de 2-oxazolinas. Los hidrogeles (HG) contuvieron grupos ácidos carboxílicos, que fueron transformados en grupos tioles, los cuales posteriormente fueron acomplejados con iones áuricos. Los iones áuricos fueron reducidos in situ y se obtuvieron nanopartículas de oro. En los hidrogeles, con y sin NP-Au, se determinó que la capacidad de absorción de agua del hidrogel se incrementa con el aumento del contenido del macromonómero en su estructura. Asimismo, ambos tipos de hidrogeles mostraron sensibilidad a la temperatura, contrayéndose su volumen al aumentar la misma. Conforme aumenta el contenido de macromonómero en el hidrogel esta contracción se obtuvo a más altas temperaturas y en mayor intensidad. La caracterización por espectroscopia UV-Visible de los HG con NP-Au mostró una absorción a $520 \mathrm{~nm}$, que es característica del plasmón de las nanopartículas de oro. La microscopia de barrido electrónico SEM mostró la presencia de nanopartículas de oro dentro de los hidrogeles en un rango de tamaños de 5 a $200 \mathrm{~nm}$.
\end{abstract}

Palabras clave: $\mathrm{N}$-isopropilacrilamida, macromonómeros, 2-oxazolinas, nanopartículas de oro, sensibilidad térmica.

\section{ELABORATION OF GOLD NANOPARTICLES IN THERMOSENSITIVES HYDROGELS}

\begin{abstract}
In this research was studied the feasibility of making gold nanoparticles within thermosensitive hydrogels which were made from $\mathrm{N}$-isopropylacrylamide and macromonomers of 2-oxazolines. The hydrogels contained carboxylic acid groups, which were transformed into thiol groups which were later complexed with auric ions. The auric ions were reduced and gold nanoparticles (NP-Au) were obtained. In hydrogels, with and without NP-Au, was determined that the water-absorbing capacity of the hydrogel increases with the increase of macromonomer content in its structure. Likewise, both hydrogels showed sensitivity to temperature, contracting its volume as it increased. As the macromonomer content in the hydrogel increases this contraction is obtained at higher temperatures and at higher intensity. The UV/Visible spectroscopy characterization of the Hydrogels with NP-Au showed a
\end{abstract}

a Pontificia Universidad Católica del Perú, Av. Universitaria 1801, Lima 32, jrueda@pucp.edu.pe

${ }^{\mathrm{b}}$ Universidad Nacional de Ingeniería. 
absorption at $520 \mathrm{~nm}$, which is characteristic of the plasmon of gold nanoparticles. The SEM electron microscopy scanning showed the presence of gold nanoparticles within the hydrogels in a range of sizes from 5 to $200 \mathrm{~nm}$.

Key words: N-isopropylacrylamide, macromonomers, 2-oxazolines, gold nanopartícles, thermoresponsive hydrogel.

\section{INTRODUCCIÓN}

Los hidrogeles son estructuras macromoleculares tridimensionales, de características hidrofílicas obtenidas a partir de polímeros sintéticos o naturales. Los hidrogeles absorben grandes cantidades de agua debido a los grupos polares que contienen en su estructura como, por ejemplo, grupos ácido carboxílicos, aminos, sulfónicos, etc. ${ }^{1,2}$. Los hidrogeles de $\mathrm{N}$-isopropilacrilamida (NiPAAm) tienen la propiedad de tener sensibilidad térmica, esto significa que, estando en un medio acuoso, se contraen cuando la temperatura del medio alcanza un determinado valor (temperatura de transición, $\mathrm{T}_{\mathrm{tr}}{ }^{2,5}$. En esta transición, la naturaleza del polímero cambia de hidrofílica a hidrofóbica. La temperatura de transición de del hidrogel de PoliNiPAAm es de aproximadamente $32^{\circ} \mathrm{C}$, la cual puede variar al copolimerizarse en NiPAAm con otros monómeros.

Las 2-oxazolinas son monómeros que pueden ser polimerizados mediante una polimerización "viva" ideal, sin reacciones secundarias, y entonces se puede controlar el peso molecular y funcionalidad de las polioxazolinas ${ }^{1,2,5-7}$.

Los materiales que consisten de nanopartículas metálicas embebidas en hidrogeles tienen potenciales aplicaciones en el campo de la catálisis, electrónica, óptica y biomedicina, entre otros. Langer y colaboradores investigaron la introducción de nanopartículas de oro en hidrogeles termosensitivos ${ }^{8,9}$. En estos hidrogeles, debido a la contracción y expansión de los mismos por el aumento o disminución de la temperatura, las nanopartículas de oro, embebidas en estos, se pueden acercar o alejar entre sí cambiando sus propiedades ópticas y electrónicas. Este cambio de propiedades los hace atractivos para determinadas aplicaciones en estos campos

El objetivo de esta investigación fue elaborar nanopartículas de oro dentro de un material sensible a la temperatura.

\section{PARTE EXPERIMENTAL}

Todas las sustancias utilizadas en esta investigación fueron adquiridas de Aldrich y purificadas según métodos estándar descritos en la literatura ${ }^{1,2,5,7}$.

Sintesis e hidrolisis del macromonómero $(M M)^{2}$ : Procedimiento: en un reactor de vidrio, bajo 
atmósfera de nitrógeno, se colocaron aproximadamente $26 \mathrm{~mL}$ de acetonitrilo, y se adicionaron 1,45 g de ioduro de sodio, 6,24 mL del monómero 2-metil-2-oxazolina (MeOXA), 3,64 mL de 2-carboxietil-2-oxazolina (EsterOXA) y $0,68 \mathrm{~mL}$ de clorometilestireno (CMS). El reactor fue cerrado, calentado a $78^{\circ} \mathrm{C}$ y agitado por 7 horas. Finalizado este tiempo, el reactor fue enfriado a temperatura ambiente y se le adicionó $0,285 \mathrm{~g}$ de $\mathrm{KOH}$ en $\mathrm{MeOH}$. El polímero fue purificado por el método de precipitación en éter dietílico. Se obtuvo aproximadamente 10,0 g de macromonómero (rendimiento del 99\%).

El macromonómero fue caracterizado por resonancia magnética nuclear protónica $\left({ }^{1} \mathrm{H}-\mathrm{RMN}\right)$ y de carbono-13 $\left({ }^{13} \mathrm{C}-\mathrm{RMN}\right)$, usando el equipo Bruker Avance III 500 NMR. Los desplazamientos químicos para el espectro ${ }^{1} \mathrm{H}-\mathrm{RMN}$, usando TMS como referente, y usando $\mathrm{CD}_{3} \mathrm{OD}$ como solvente son: $\delta$ : 2,11 ( $\left.\mathrm{CH}_{3}\right) ; 2,5-2,8\left(\mathrm{COCH}_{2} \mathrm{CH}_{2} \mathrm{COO}-\right) ; 3,53\left(\mathrm{NCH}_{2} \mathrm{CH}_{2}\right)$; $3,67-3,71\left(\mathrm{COOCH}_{3}\right) ; 4,66\left(\mathrm{Ar}_{-} \mathrm{CH}_{2}\right) ; 5,21-5,3$ y 5,7-5,9 $\left(\mathrm{CH}_{2}=\right) ; 6,6-6,8(\mathrm{CH}=) ; 7,1-7,5$ $\left(\mathrm{H}_{\text {AROMÁtICOS }}\right)$.

Los desplazamientos químicos para el espectro ${ }^{13} \mathrm{C}-\mathrm{RMN}$ (en $\mathrm{CD}_{3} \mathrm{OD}$ ) son: $\delta: 21,3-21,8$ $\left(\mathrm{CH}_{3}\right) ; 28,5\left(-\mathrm{COCH}_{2}\right) ; 30\left(\mathrm{CH}_{2} \mathrm{COCH}_{3}\right) ; 44-48\left(\mathrm{NCH}_{2} \mathrm{CH}_{2}\right) ; 52,2\left(\mathrm{COOCH}_{3}\right) ; 54,1(\mathrm{Ar}-$ $\left.\mathrm{CH}_{2}\right) ; 61\left(\mathrm{CH}_{2} \mathrm{OH}\right) ; 115\left(\mathrm{CH}_{2}=\right) ; 125-131$ ( $\left.\mathrm{C}_{\text {ARomáticos }}\right), 137-140$ ( $\left.\mathrm{C}_{\text {AROMÁticos }}\right) ; 137,5$ $(-\mathrm{CH}=) ; 173,6\left(\mathrm{COCH}_{3}+\mathrm{COCH}_{2}^{-}\right) ; 175,1\left(\mathrm{COOCH}_{3}\right)$.

El macromonómero fue hidrolizado con una solución de hidróxido de sodio. A un balón de $250 \mathrm{~mL}$, se agregó 6,0 g de macromonómero MM con $160 \mathrm{~mL}$ de $\mathrm{NaOH} 0,1 \mathrm{~N}$, este sistema se dejó bajo agitación y calentamiento, a $45^{\circ} \mathrm{C}$ por 7 horas. Terminado este tiempo, el sistema fue enfriado y luego, el sistema fue neutralizado con $\mathrm{HCl}$ diluido, hasta llegar a un $\mathrm{pH}$ de $5,6^{2}$.

El macromonómero se purificó por diálisis (membranas Zellu Trans Carl Roth GmbH, MWCO: 1000), para lo cual, el macromonómero, disuelto en agua, fue colocado dentro de las membranas, y estas fueron sumergidas en agua destilada durante 48 horas, tiempo durante el cual se cambió 4 veces el agua en la que estaba sumergida la membrana. Finalmente se extrajo la membrana y el agua de la solución del macromonómero fue eliminada por liofilización.

El macromonómero hidrolizado $(\mathrm{MMH})$ fue caracterizado estructuralmente por sus espectros de resonancia magnética nuclear protónica y de carbono -13. La diferencia con el espectro del macromonómero original fue que desaparecieron las señales a 3,6 ppm y 52 ppm en los espectros ${ }^{1} \mathrm{H}-\mathrm{RMN}$ y ${ }^{13} \mathrm{C}-\mathrm{RMN}$, respectivamente que corresponden al grupo metilo del éster.

Síntesis de los hidrogeles y su posterior modificación ${ }^{2}$ : Procedimiento típico (Hidrogel HG1): En un vaso de $50 \mathrm{~mL}$, se mezclaron $0,5 \mathrm{~g}$ de $\mathrm{N}$-isopropilacrilamida (NiPAAm), 0,08 $\mathrm{g}$ de macromonómero hidrolizado (MMH), 0,030 g de N,N'-metilenbisacrilamida (MBIS), $0,030 \mathrm{~g}$ de tetrametiletilendiamina (TEMED) y $5 \mathrm{~mL}$ de agua hasta disolución total. A esta mezcla se le burbujeó nitrógeno durante 5 minutos. El sistema fue cerrado y colocado en un baño a $5^{\circ} \mathrm{C}$ durante 10 minutos. En forma paralela, en otro vaso de $25 \mathrm{~mL}$ se disolvió $0,010 \mathrm{~g}$ de persulfato de amonio (APS) en $1 \mathrm{~mL}$ de agua y se enfrió a $5^{\circ} \mathrm{C}$ y se burbujeó 
nitrógeno. A continuación, a la primera mezcla se le agregó la solución de APS y se mantuvo la temperatura de la mezcla a $5^{\circ} \mathrm{C}$. En 15 minutos ocurrió la formación del hidrogel.

El hidrogel se purificó de la siguiente manera: en un litro de agua destilada se colocó una porción del hidrogel por 48 horas para eliminar restos de monómeros y de iniciador que no hubieran reaccionado, durante este período el agua fue cambiada cuatro veces. Finalizada la purificación, el hidrogel fue secado a $32^{\circ} \mathrm{C}$ hasta alcanzar un peso constante, luego fue guardado en un desecador. Este hidrogel fue denominado HG1, y se obtuvo 0,61 $\mathrm{g}$ (rendimiento de 98,70\%). Para la síntesis y purificación de los hidrogeles HG2 y HG3, se usó el mismo procedimiento.

Modificación de los hidrogeles con grupos tioles: 0,40 g del hidrogel HG1 hidrolizado se colocó en $10 \mathrm{~mL}$ de agua destilada a $10{ }^{\circ} \mathrm{C}$. A esta mezcla se le agregó $0,079 \mathrm{~g}$ de 1-etil-3-(3-dimetilaminopropil) carbodiimida (EDAC) y 0,088 g de sal sódica de N-hidroxisulfosuccinimida (NHS-Na). Se dejó en reposo esta mezcla durante seis horas. Luego, se agregó a la mezcla anterior $0,050 \mathrm{~g}$ de hidrocloruro de cisteamina (en exceso estequiométrico). Se dejó reaccionar esta mezcla por cuatro días en oscuridad y a $5^{\circ} \mathrm{C}$. Luego, el hidrogel fue lavado con agua destilada hasta $\mathrm{pH}$ neutro. El hidrogel se secó y guardó en un desecador hasta su utilización.

Elaboración de nanopartículas de oro dentro del hidrogel modificado: El hidrogel $(0,25 \mathrm{~g})$ se colocó en $30 \mathrm{~mL}$ de una solución de ácido tetracloroáurico $\left(0,0188\right.$ gramos de $\mathrm{H}\left[\mathrm{AuCl}_{4}\right]$ en $200 \mathrm{~mL}$ de agua destilada) por dos días, al cabo de los cuales el hidrogel estaba embebido de esta solución y había tomado una fuerte coloración amarilla. Los iones áuricos fueron reducidos con $25 \mathrm{ml}$ de una solución que contenía $0,25 \mathrm{~g}$ de citrato de trisódico en $50 \mathrm{~mL}$ de agua $^{10}$. Como producto de la aplicación de este procedimiento se obtuvieron nanopartículas de oro dentro de los hidrogeles y visualmente se pudo notar como el hidrogel inicialmente de color amarillo adquirió un color violeta oscuro.

Prueba de absorción de agua: Se colocó en un vaso una porción de hidrogel hidrolizado seco (peso inicial=Ws) en $80 \mathrm{~mL}$ de agua de destilada a temperatura ambiente durante 24 horas. Pasado este tiempo, se obtuvo el peso del hidrogel hinchado en agua (Wh). El grado de absorción de agua del hidrogel fue calculado mediante la ecuación:

$$
\mathrm{Q}=(\mathrm{Wh}-\mathrm{Ws}) / \mathrm{Ws}
$$

donde Q es el grado de absorción de agua (g H2O/g de HG seco).

Prueba de sensibilidad a la temperatura: Se determinó la contracción del volumen de los hidrogeles en función de su pérdida de peso y se asumió que el peso es proporcional al volumen. La masa a $25^{\circ} \mathrm{C}$ fue relacionado con el volumen inicial del hidrogel. El porcentaje de contracción, en respuesta al aumento de temperatura, se determinó mediante la ecuación:

$$
\text { Porcentaje de contracción }=100 *\left(\mathrm{~W}_{\text {inicial }}-\mathrm{W}_{\text {final }}\right) / \mathrm{W}_{\text {inicia }} \ldots \ldots . . .(2)
$$


Procedimiento: En un vaso se colocó una porción del hidrogel en $80 \mathrm{~mL}$ de agua a temperatura ambiente por 24 horas. Pasado este tiempo, se sacó el hidrogel del agua y se determinó su peso $\left(\mathrm{W}_{\text {inicial }}\right)$. Seguidamente, la misma porción de hidrogel se sumergió nuevamente en agua y fue colocado en la estufa a una temperatura determinada durante 40 minutos; luego, el hidrogel nuevamente se sacó del agua y se pesó $\left(\mathrm{W}_{\text {final }}\right)$. Se determinó el peso remanente del hidrogel a esta temperatura mediante la ecuación:

$$
\text { Porcentaje de peso remanente }=100 *\left(\mathrm{Peso}_{\text {final }} / \mathrm{Peso}_{\text {inicial }}\right)
$$

La contracción es la diferencia entre la masa inicial y la masa remanente. Para cada temperatura, se obtuvo un porcentaje de peso remanente y estos valores fueron graficados versus la temperatura $\left({ }^{\circ} \mathrm{C}\right)$.

Análisis espectroscópico UV/Visible de los hidrogeles: Se colocó una muestra de hidrogel (0,5 g) en una celda dentro del espectrofotómetro Ultravioleta/Visible, marca Thermo Electron Corporation, Helios Gamma. Se usó un baño termostatizado con agua destilada para calentar la muestra mediante una chaqueta de calentamiento; las mediciones se iniciaron a $25^{\circ} \mathrm{C}$, con incrementos de $4^{\circ} \mathrm{C}$ cada 20 minutos.

Análisis por microscopía de barrido electrónico (SEM): Las muestras fueron analizadas por la Ing. Kristina Schelestow en la Universidad Tecnológica de Ilmenau en Alemania, aplicando el procedimiento estándar.

\section{RESULTADOS Y DISCUSIÓN}

La estrategia seguida fue la siguiente: se elaboró un hidrogel de poliNiPAAm que es sensible a la temperatura y el cual contenía grupos ácido carboxílicos (provenientes del macromonómero hidrolizado de las 2-oxazolinas) que podrían ser modificados en grupos tioles, los cuales acomplejarían los iones áuricos y por posterior reducción se formarían las NP-Au dentro del hidrogel.

Este nuevo material mantendría a las nanopartículas de oro distribuidas por todo el hidrogel, el cual al contraerse, por un aumento de la temperatura, acercaría a las nanopartículas de oro confiriéndoles a estas nuevas propiedades físicas.

En una publicación anterior ya hemos descrito con todo detalle la síntesis y caracterización del macromonómero y del hidrogel que han sido usados en esta investigación ${ }^{2}$. Por lo que mencionaremos este aspecto brevemente y nos centraremos más en la elaboración de las nanopartículas de oro en los hidrogeles.

Síntesis del macromonómero: La síntesis fue realizada mediante la polimerización catiónica por apertura de anillo de la 2-metil-2-oxazolina (MeOXA) y la 2-carboxietil-2-oxazolina (EsterOXA) iniciada por el clorometilestireno (CMS) en presencia del catalizador ioduro 
de sodio (esquema 1). La reacción se realizó a una temperatura relativamente baja, de $78^{\circ} \mathrm{C}$, durante un tiempo también relativamente breve ( 7 horas) y con una baja concentración del iniciador $([\mathrm{CMS}]=0,13 \mathrm{M})$ en el medio de reacción para evitar así una polimerización prematura del grupo vinilo del CMS. Sin embargo, estas condiciones de reacción sí fueron suficientes para conseguir la polimerización cuantitativa de las 2-oxazolinas. Se obtuvo un macromonómero de grado de polimerización de 20 unidades (peso molecular de $2220 \mathrm{~g} / \mathrm{mol}$, obtenido por RMN) y con un contenido de Esteroxa del $25 \%$ molar. El macromonómero fue hidrolizado con una solución de hidróxido de sodio y luego neutralizado con ácido clorhídrico. Tanto el macromonómero original como el hidrolizado fueron caracterizados por RMN. Se pudo observar que las señales a $3,67 \mathrm{ppm}\left({ }^{1} \mathrm{H}-\mathrm{RMN}\right)$ y $52,2 \mathrm{ppm}\left({ }^{13} \mathrm{C}-\mathrm{RMN}\right)$ del grupo metilo del éster desaparecieron después de la hidrólisis, demostrando que ocurrió una hidrólisis completa de todos los grupos éster del copolímero. Sólo se produjo la hidrólisis de los grupos éster sin afectar a los grupos amida ni a los grupos vinilos debido a las condiciones "suaves" del procedimiento de hidrólisis.

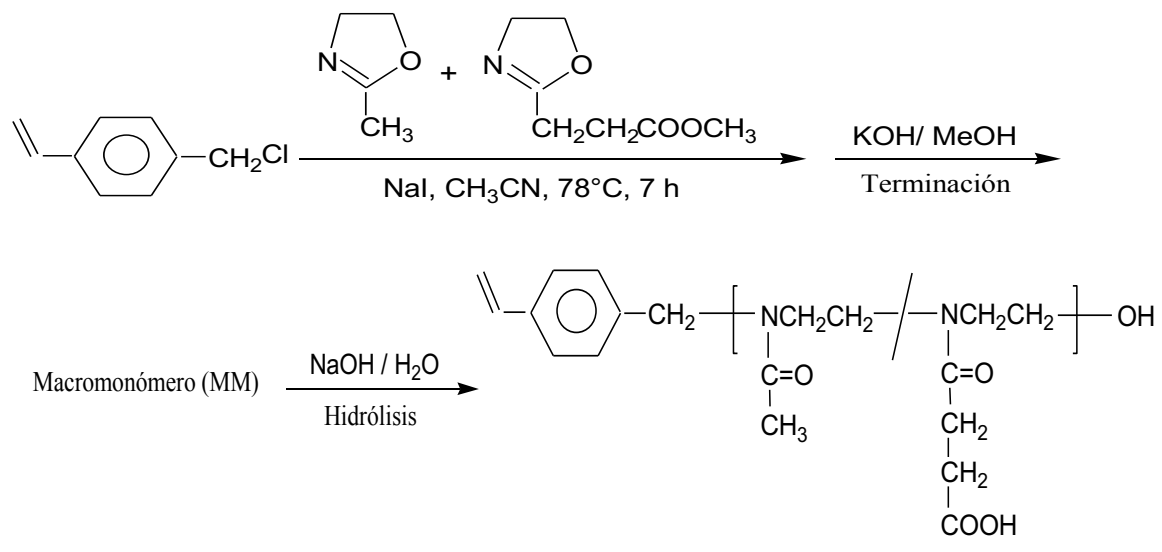

Macromonómero (MMH)

Esquema 1. Síntesis del macromonómero

Síntesis de los hidrogeles: Los hidrogeles fueron obtenidos mediante la copolimerización por radicales libres de la $\mathrm{N}$-isopropilacrilamida (NiPAAm), y el macromonómero hidrolizado de 2-oxazolina en presencia de N,N'-metilenbisacrilamida (MBIS). La polimerización se realizó en medio acuoso y fue iniciada por un sistema de iniciación redox basado en persulfato de amonio (APS) y catalizador N,N,N',N'-tetrametiletilendiamina (TEMED) (esquema 2). Los detalles experimentales y resultados obtenidos en la síntesis de los hidrogeles se resumen en la tabla 1 . 
Tabla 1. Síntesis de hidrogeles (datos experimentales y resultados).

\begin{tabular}{|c|c|c|c|c|c|c|c|}
\hline Hidrogel & $\begin{array}{c}\text { NiPAAm } \\
\mathbf{( g )}\end{array}$ & $\begin{array}{c}\text { NiPAAm } \\
(\mathbf{m m o l})\end{array}$ & $\begin{array}{c}\mathbf{M M H}^{\mathbf{b}} \\
\mathbf{( g )}\end{array}$ & $\begin{array}{c}\text { MMH } \\
(\mathbf{m m o l})\end{array}$ & $\frac{\text { NiPAAm }^{\mathbf{m}}}{\mathbf{M M H}}$ & $\begin{array}{c}\text { Masa HG } \\
\text { obtenido } \\
\mathbf{( g )}\end{array}$ & $\mathbf{R}^{\mathbf{d}} \mathbf{( \% )}$ \\
\hline HG0 & 0,50 & 4,42 & 0 & 0 & - & 0,53 & 99,1 \\
\hline HG1 & 0,50 & 4,42 & 0,080 & 0,199 & 22 & 0,61 & 98,7 \\
\hline HG2 & 0,50 & 4,42 & 0,040 & 0,099 & 45 & 0,57 & 97,6 \\
\hline HG3 & 0,50 & 4,42 & 0,015 & 0,037 & 119 & 0,54 & 98,5 \\
\hline
\end{tabular}

Donde:

a: N-isopropilacrilamida (NiPAAm).

b: Macromonómero (MMH) de MeOXA ( $75 \%$ molar) y EsterOXA ( $25 \%$ molar).

c: Relación molar de monómeros de NiPAAm/MM.

d: Rendimiento $(\%)=100 *$ (Peso HG obtenido/Suma de pesos de reactantes (NiPAAm, macromonómero y N,N'-metilenbisacrilamida (MBIS).

Se elaboraron cuatro hidrogeles denominados HG0, HG1, HG2, y HG3. El HG0 no contuvo macromonómero y los hidrogeles HG-1, 2 y 3 tuvieron cantidades decrecientes de macromonómero en su estructura.

\section{Modificación del hidrogel y obtención de nanopartículas de oro en su estructura}

Los hidrogeles fueron parcialmente modificados en su estructura química mediante la introducción de grupos tioles en la misma. Esto se logró mediante la reacción de los grupos ácido carboxílicos del hidrogel con el hidrocloruro de cisteamina, reacción que fue promovida mediante el uso del EDAC y la sal sódica de la hidroxisuccinimida (NHS-Na) (esquema 3). Los grupos tioles introducidos al hidrogel sirvieron para acomplejar los iones áuricos, que posteriormente fueron reducidos in situ con citrato trisódico para obtener nanopartículas de oro $(\mathrm{NP}-\mathrm{Au})$.

Prueba de absorción de agua: La capacidad de absorción de agua para los hidrogeles sintetizados fue de 9,$17 ; 12,8 ; 11,3 ; \mathrm{y} 10,7$ (g de agua/ $\mathrm{g}$ de hidrogel seco) para los hidrogeles HG0, HG1, HG2 y HG3, respectivamente. Se observa que esta absorción aumenta al incrementarse el contenido de macromonómero en el hidrogel. Probablemente esto se debe al aumento del contenido de grupos polares (ácidos carboxílicos del macromonómero) lo que incrementa la polaridad del hidrogel. 


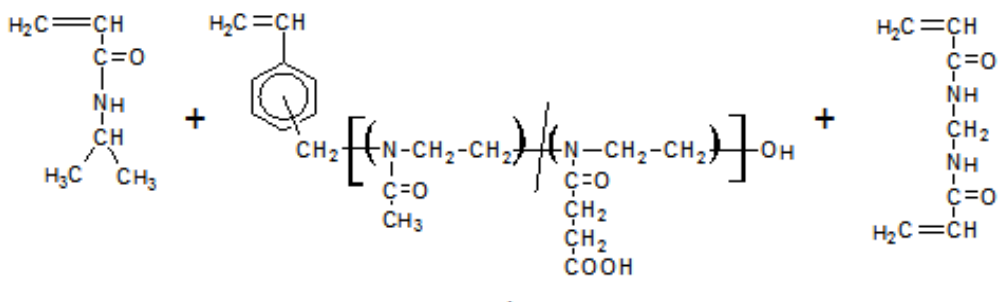

NiPAAm

Macromonómero de 2- axazolina

MBIS
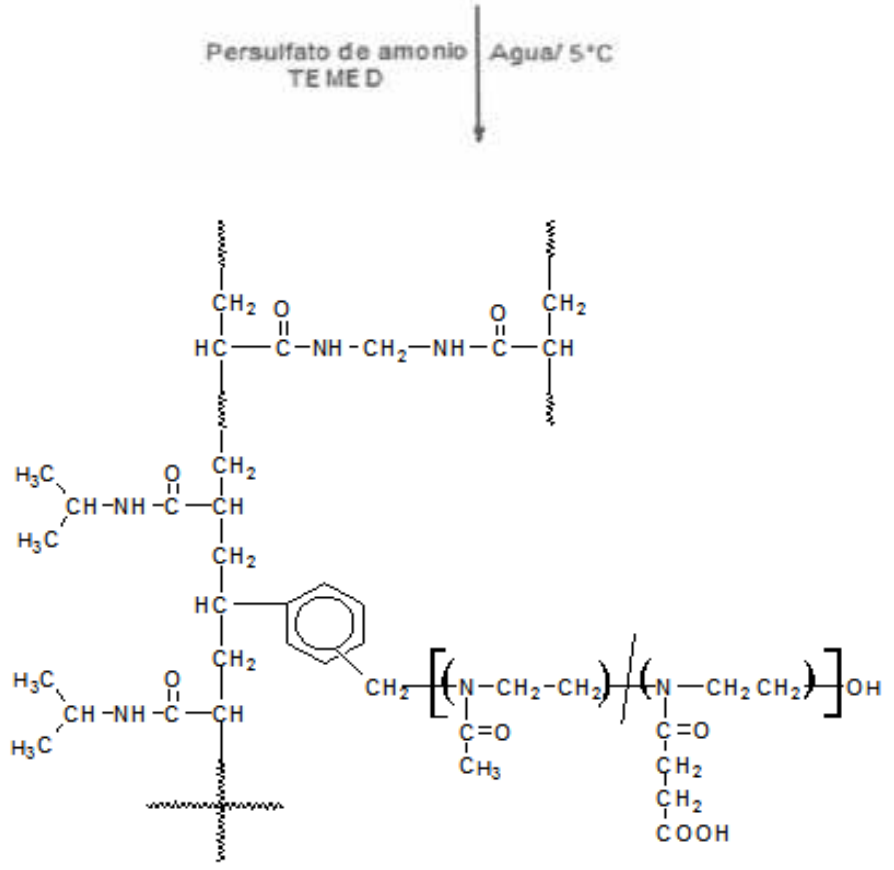

Hidrogel

Esquema 2. Síntesis de hidrogeles.

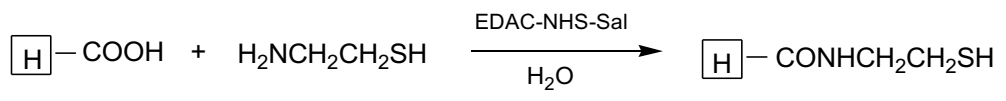

(continúa la reacción)... 


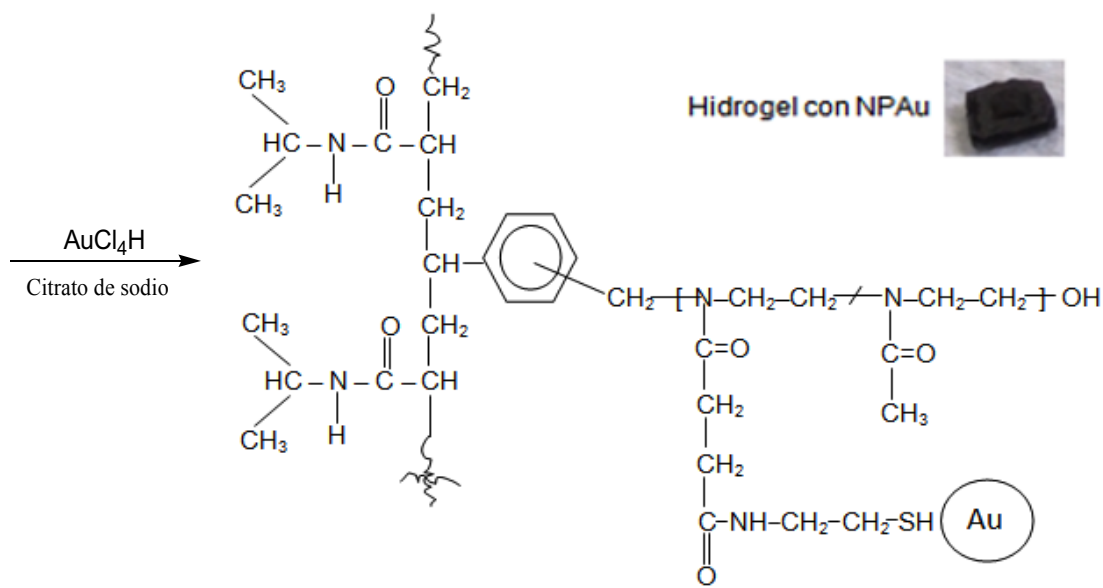

Esquema 3. Elaboración de nanopartículas de oro en los hidrogeles

Prueba de sensibilidad a la temperatura de los hidrogeles: tanto los hidrogeles originales como los modificados con nanopartículas de oro presentaron sensibilidad a la temperatura. Esta sensibilidad se expresó como una contracción del volumen del hidrogel, saturado en agua, al aumentar la temperatura. Debido a la forma irregular del hidrogel no fue posible medir su volumen, pero se midió el peso de dicho hidrogel $\left(\mathrm{W}_{\text {inicial }}\right)$ y se asumió que este peso es proporcional al volumen del mismo. Así, la disminución de peso que sufre el hidrogel, por la pérdida de una fracción del agua absorbida, al aumentar la temperatura del medio, se tomó como el equivalente a una contracción del volumen del mismo. El porcentaje de peso remanente se calculó mediante la ecuación (3), mencionada anteriormente.

Las figuras 1 y 2 muestran los gráficos de la temperatura versus porcentaje de peso remanente para todos los hidrogeles sin NP-Au y con NP-Au.

En todos los experimentos realizados, los hidrogeles (HG) tuvieron una misma cantidad de NiPAAm y MBIS, y solo la cantidad de macromonómero hidrolizado ( $\mathrm{MMH}$ ) fue variada. De la figura 1 se observa a mayor cantidad de macromonómero dentro del hidrogel la contracción es mayor (p. ej. comparar HG1 vs HG2). Inclusive se observa que el HG sin contenido de macromonómero se contrae menos en comparación a los otros. Este hecho se debe posiblemente que a mayor cantidad de macromonómero $(\mathrm{MMH})$ contenido en el hidrogel (HG) hay una mayor cantidad de grupos $\mathrm{COOH}$ y $\mathrm{COONa}$, lo cual aumenta su polaridad y se forma una mayor cantidad de enlaces tipo puente de hidrógeno entre estos y los grupos polares amida de los segmentos de poliNiPAAm. Así, al ocurrir la transición conformacional el mayor número de interacciones puente de hidrógeno hará colapsar, en mayor medida, el hidrogel. Se observa también que la Ttr aumenta al incrementarse el contenido de macromonómero en el hidrogel por la mayor polaridad de la estructura. 


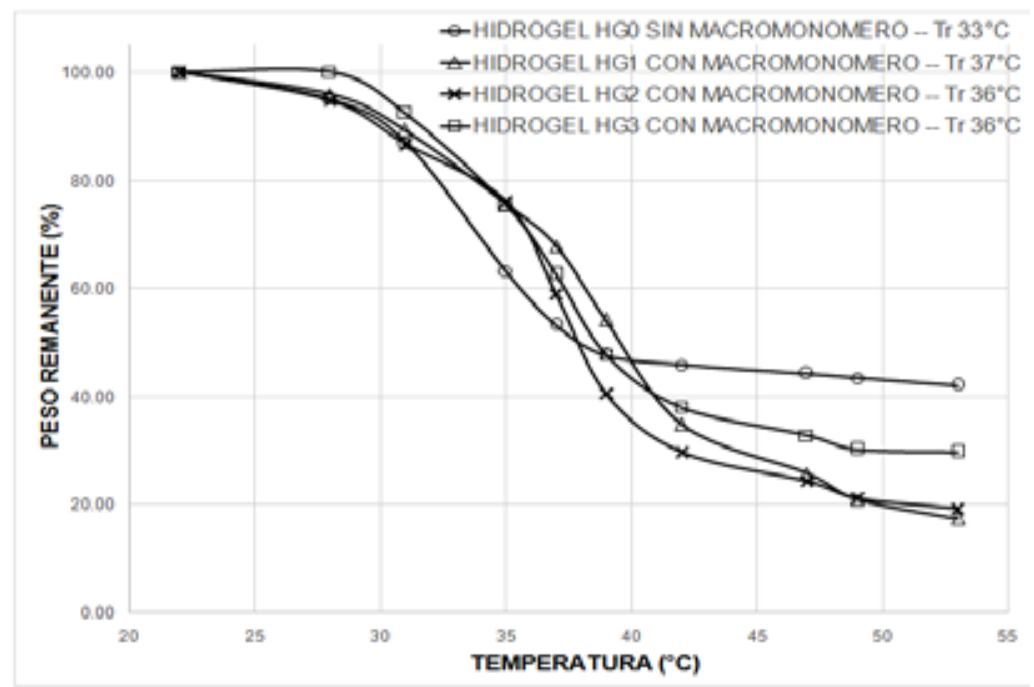

Figura 1. Temperatura $\left({ }^{\circ} \mathrm{C}\right)$ versus Peso remanente $(\%)$ de los hidrogeles sin NP-Au.

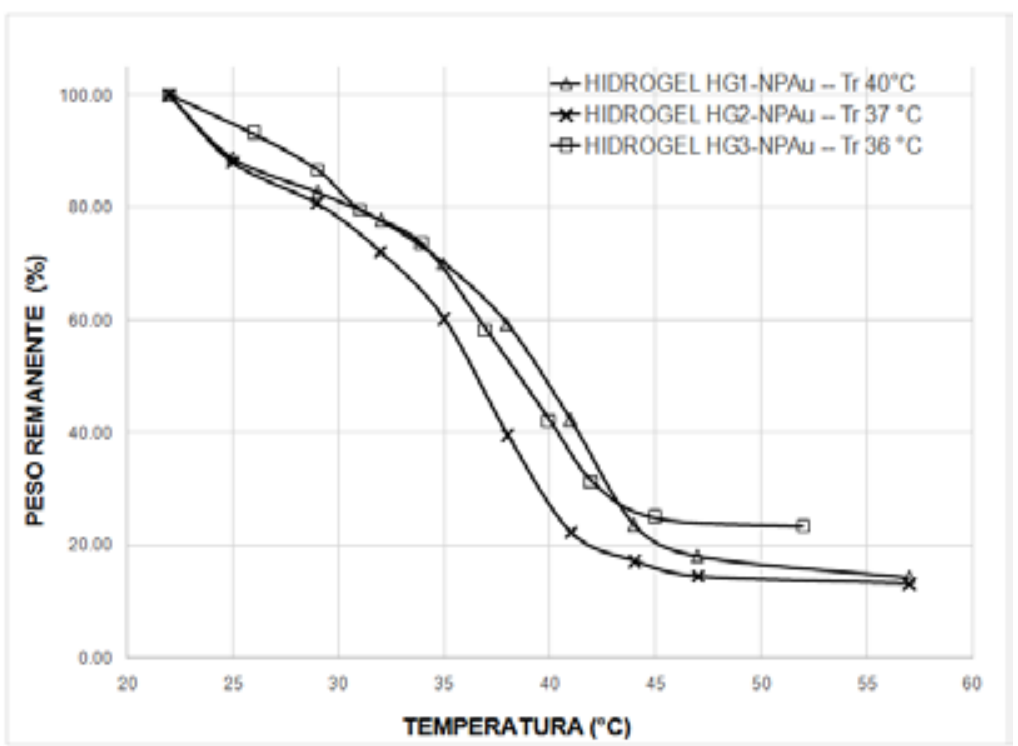

Figura 2. Temperatura $\left({ }^{\circ} \mathrm{C}\right)$ versus Peso remanente $(\%)$ de los hidrogeles con NP-Au. 
En el caso de los HG conteniendo NP-Au se observa (Fig. 2) que su contracción es mayor que sus homólogos con NP-Au. Además que su temperatura de transición aumenta ligeramente. Esto podría explicarse debido a que las NP-Au actuarían como puntos de unión o de encuentro de las cadenas del poliNiPAAm cuando ocurre la transición térmica. Las cadenas polares de poliNipAAm y de macromonómero estarían absorbidas en la superficie de la NP-Au que es relativamente más grande que las cadenas de poliNiPAAm y al colapsarse la estructura esto conduciría a una mayor compactación de la misma por la mayor cercanía de las cadenas de poliNiPAAm que colapsan.

Análisis espectroscópico UV/Visible: Se realizó el análisis por espectroscopia UV/Visible de los hidrogeles HG1, HG2, HG3 con y sin nanopartículas de oro a diferentes temperaturas. En la figura 3 se puede observar que el hidrogel HG1-Au presenta un pico cerca de $520 \mathrm{~nm}$ el cual no cambia conforme se eleva la temperatura del medio, la presencia del plasmón típico de las nanopartículas de oro cerca de $520 \mathrm{~nm}$ es un claro indicador de la presencia de estas en el hidrogel. El mismo plasmón fue observado en los hidrogeles HG2 y HG3.

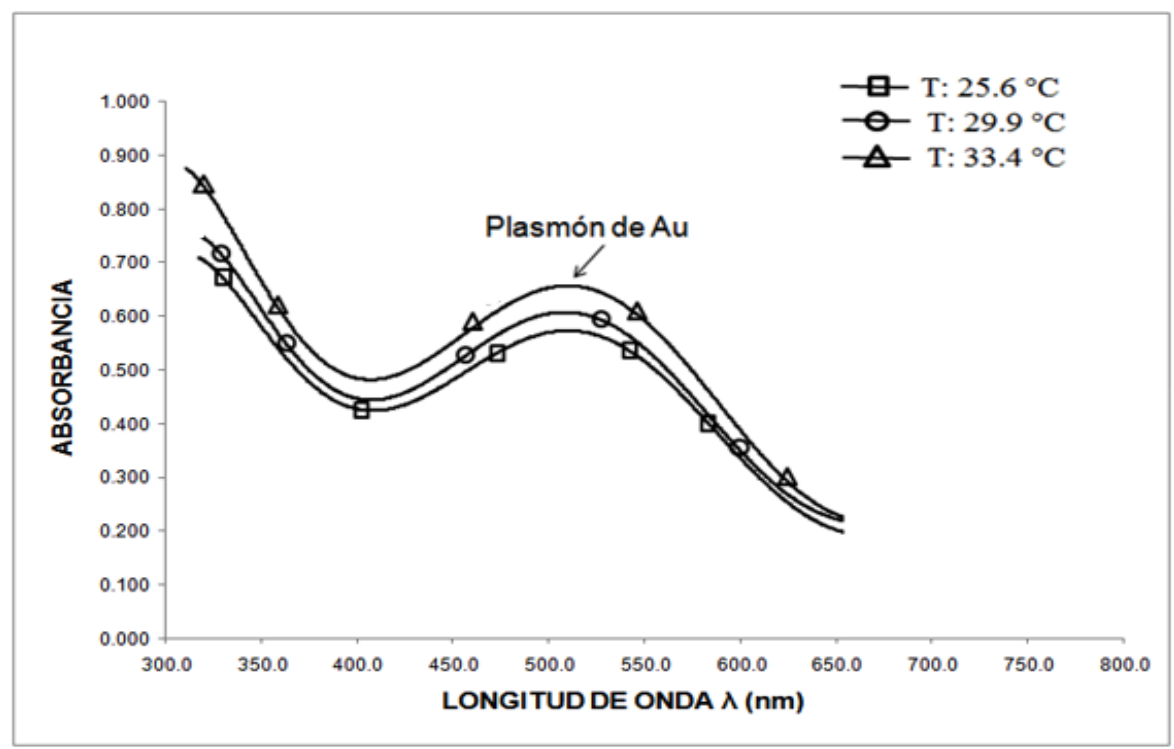

Figura 3. Absorbancia vs longitud de onda $\lambda(\mathrm{nm})$ del hidrogel HG1-Au: el espectro UV/Vis de los hidrogeles que no contenían NP-Au no mostró el plasmón característico a $520 \mathrm{~nm}$ y también su absorbancia fue menor.

Obtención y análisis de las imágenes por microscopía de barrido electrónico (SEM):

Se hizo un análisis por SEM a los hidrogeles que contenían NP-Au. A modo de ejemplo, en la figura 4 se muestra la microfotografía electrónica CEM del hidrogel HG1 con NP-Au. Los tamaños de las mismas oscilaron, en todos los casos, entre 5 y $200 \mathrm{~nm}$. 


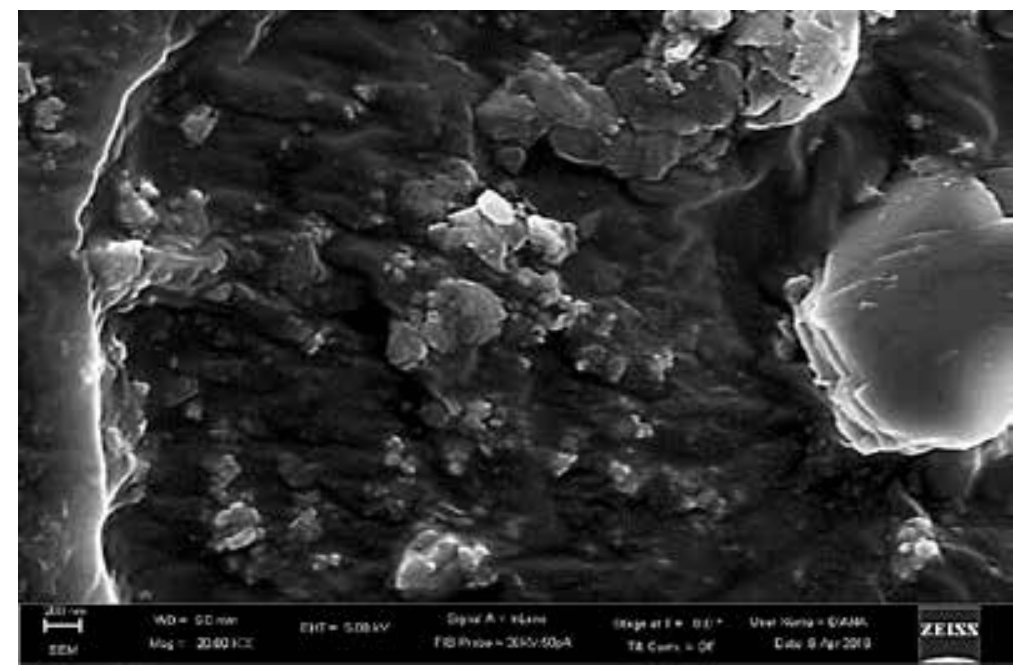

Figura 4. Imagen SEM del Hidrogel HG1 con NP-Au

El estudio de las propiedades ópticas y electrónicas de las NP-Au en los hidrogeles se realizará en un estudio posterior.

\section{CONCLUSIONES}

Fue posible la obtención de nanopartículas de oro (NPAu) in situ en hidrogeles de NiPAAm y macromonómero de 2-oxazolina, mediante el acomplejamiento de los iones aúricos con los grupos tioles del hidrogel y su posterior reducción. Se demostró la obtención de las NPAu mediante la espectrometría UV-Visible (plasmón a $520 \mathrm{~nm}$ ) y la microscopía de barrido electrónico (SEM), los tamaños de las NP-Au oscilaron entre 5 y $200 \mathrm{~nm}$.

Al aumentar el contenido de macromonómero en el hidrogel aumenta la absorción de agua y también la temperatura de transición conformacional del hidrogel. Además, el hidrogel sufre una mayor contracción al incrementarse el contenido de macromonómero. Esto podría deberse al carácter fuertemente polar del macromonómero, lo que incrementa fuertemente la hidrofilicidad del hidrogel y se formarían más interacciones puentes de hidrógeno entre los grupos ácido carboxílicos del macromonómero y los grupos amida del poliNiPAAm.

Con la presencia de NP-Au en los hidrogeles, aumenta ligeramente la temperatura de transición conformacional y los hidrogeles se contraen aún más en comparación con sus homólogos sin NP-Au. Este fenómeno puede deberse a que las NP-Au podrían actuar como elementos de unión o de acercamiento entre los segmentos de poliNiPAAm que colapsarían en mayor grado al ocurrir la transición conformacional. 


\section{REFERENCIAS BIBLIOGRÁFICAS}

1. Rueda J, Komber H, Voit B, Cedrón J, Shevtsova G. Synthesis of New Hydrogels by Copolymerization of Poly(2-methyloxazoline) bis (macromonomers) and N-vinylpyrrolidone. Macromol Chem Phys. 2003; 204: 947-953.

2. Rueda J, Campos E, Komber H, Zschoche S, Voit B. Synthesis and Characterization of New $\mathrm{pH}$ and Thermoresponsive Hydrogels Based on N-Isopropylacrylamide and 2-oxazolines. Des Monomers Polym. 2014; 17: 208-216.

3. Schild H. Poly(N-isopropylacrylamide): Experiment, Theory and Application. Prog Polym Sci. 1992; 17: 163-240.

4. Di mitrov I, Trzebicka B, Müller AHE, Dworak A, Tsvetanov C. Thermosensitive WaterSoluble Copolymers with Doubly Responsive Reversibly Interacting Entities. Prog Polym Sci. 2007; 32: 1278-1317.

5. Zschoche S, Rueda J, Binner M, Komber H, Janke A, Voit B. Reversibly Switchable pH and Thermoresponsible Core-Shell Nanogels based on Poly (NiPAAm)-graft-poly(2carboxyethyl-2-oxazoline)s. Macromol Chem Phys. 2012; 213: 215-216.

6. Aoi K, Okada M. Polymerization of Oxazolines. Prog Polym Sci. 1996; 21: 151-208.

7. Rueda J, Asmad M, Ruiz V, Komber H, Zschoche S, Voit B. Synthesis and characterization of new bi-sensitive copoly (2-oxazolines). Des Monomers Polym. 2015; 18: 761-768.

8. Wang Ch, Flynn NT, Langer R. Controlled Structure and Properties of Thermoresponsive Nanoparticle-Hydrogel Composites. Adv Mater. 2004; 16: 1074-1079.

9. Pong FY, Lee M, Bell JR, Flynn NT. Thermoresponsive Behavior of Poly(NIsopropylacrylamide) Hydrogels Containing Gold Nanostructures. 2006; 22 (8): 38513857

10. Corzo A. Síntesis de nanopartículas de oro obtenidas por reducción de [HAuCl4]. Rev Soc Quím Perú. 2012; 78(2): 79-90. 